\title{
CIRED 2021: Bericht über die Session 5 - Planning of Power Distribution Systems
}

\section{R. Braunstein OVE}

angenommen am 19. Oktober 2021, online publiziert am 8. November 2021 (c) Springer-Verlag GmbH Austria, ein Teil von Springer Nature 2021
Für die diesjährige CIRED-Session 5 - Verteilnetzplanung wurden rund 240 Kurzfassungen eingereicht, wovon nach Begutachtung und Übermittlung der Langfassungen 143 Arbeiten veröffentlicht wurden. Die hohe Zahl an Einreichungen spiegelte die Aktualität der Analyse, Planung und Weiterentwicklung von Verteilernetzen wider. Von den 143 veröffentlichten Arbeiten wurden 24 in der MainSession präsentiert. Auch das Research and Innovation Forum (RIF) bot fünf Forscher/innen die Gelegenheit, ihre Arbeiten vorzustellen. Der Großteil der Autor/innen präsentierte seine Arbeiten auch in Form eines Posters. Die CIRED wurde heuer erstmalig als Webkonferenz abgehalten. Klassisches Socializing fand nicht statt, es gab jedoch einen Austausch über die Online-Fragestellungen des Auditoriums. Die CIRED-Onlineplattform kann als gelungen bezeichnet werden. Positiv hervorzuheben ist die On-Demand-Funktion, die ein nachträgliches Ansehen der Sessions ermöglicht.

Wie in der Vergangenheit wurde die Main-Session 5 in folgende thematische Sessions unterteilt:

\section{- Main-Session 5.1: Risikoabschätzung und Asset-Manage- ment}

In der Main-Session 5.1 wurden sechs Beiträge präsentiert, zwei davon stammten aus Österreich.

In einem Beitrag aus Frankreich (468) wurde auf die Resilienz des Verteilernetzes im Hinblick auf die klimatischen Veränderungen eingegangen. Die Simulationen bis 2050 verdeutlichten das zukünftig verstärkte Aufkommen von Windstürmen, schweren Regenfällen, zunehmend hohen Temperaturen und Waldbränden. Auf Basis der durchgeführten Simulationen wurden Maßnahmen für den Verteilernetzbetreiber, wie Umstrukturierung der Netze in Flutzonen, verstärkte Digitalisierung und Verbesserung des Krisenmanagements, eingeführt.

Ein österreichischer Beitrag (392, Kienberger et al.) widmete sich der Power-to-Gas-Sektorenkopplung als Alternative zum klassischen, durch eine Vielzahl von erneuerbaren Einspeiseanfragen bedingten Netzausbau im 110-kV-Netz.

In einem weiteren österreichischen Beitrag (450, Fuchs et al.) wurde ein Tool zur techno-ökonomischen Bewertung von hybriden AC/DC-Niederspannungsteilnetzen vorgestellt. Die Autorin wurde mit dem "Best Young Academic Paper Award" der Session 5 ausgezeichnet.

\section{- Main-Session 5.2: Netzentwicklung}

Die Main-Session 5.2 widmete sich der Aufgabenstellung, wie zukünftig dezentrale Erzeugungseinheiten und Elektromobilität mit minimal nötigem Investitionsaufwand ins Verteilernetz integriert werden können.

Die Session wurde vom Begriff der "Non-Wire Alternatives" (NWA) geprägt. Vielfach lassen sich kostenintensive Netzinvestitionen durch ein Zusammenspiel von Speichermaßnahmen und intelligenten Regelungen, wie Spannungsreglung, Flexibilitätsanreize, gesteuertes Laden etc., verzögern. Dieser Tenor zog sich durch die ganze Session und wurde übrigens auch intensiv im Round Table 5 - "Digital DSO and Flexibility in Active Distribution Networks" diskutiert, eine Anpassung des regulatorischen Rahmens ist jedoch erforderlich.

In diesem Zusammenhang sei ein Überblickbeitrag aus den USA (583) besonders erwähnenswert.

In einem Beitrag aus Deutschland (722) wurde die Vermaschung eines Mittelspannungsnetzes aufgrund verstärkter Einspeiseanfragen als Alternative zum klassischen Netzausbau vorgestellt.

\section{- Main-Session 5.3: Verteilnetzplanung}

Die Main-Session 5.3 konzentrierte sich schwerpunktmäßig auf die zukünftige Rolle von Flexibilitäten in Bezug auf die Verteilernetzplanung und Netzentwicklung. Es wird in diesem Zusammenhang nur mehr selten vom "Prosumer", sondern vom "Active Consumer" und auch vom "Active DSO" gesprochen.

Im Zuge der Session kam es zur Diskussion über Flexibilitätsanbieter bzw. zur Fragestellung, wer der "Asset Owner" von Speichern idealerweise sein sollte. Grundsätzlich muss der DSO bei diesen Fragen in hohem Maße eingebunden sein, da dessen Vorgaben, beispielsweise zur Speicherdimensionierung der Standortwahl, und auch die vorherrschende Netztopologie wesentlich sind. Ansonsten besteht die Gefahr, dass die untersuchten Flexibilitäten (Einspeiseverhalten, Lastverhalten, Speicher etc.) reinen Marktsignalen folgen und dass die eigentümliche Idee, Netzinvestitionen hintenanzustellen und mehr erneuerbare Erzeuger, Elektromobilität und Wärmepumpen integrieren zu können, aufgrund von rein marktdienlichem Verhalten nicht zur Umsetzung gelangt.

Passend dazu sei auch die Rolle des DSO beim Ausbau der öffentlichen Ladeinfrastruktur erwähnt. In einem schottischen Beitrag (1140) wurden anhand eines Pilotprojektes die Vorteile der DSO-getriebenen Standortwahl für öffentliche Ladeinfrastruktur angeführt und wirtschaftlich bewertet.

\section{- Main-Session 5.4: Tools und Methoden}

Die Main-Session 5.4 befasst sich traditionell mit neuentwickelten Werkzeugen und Berechnungsmodellen, die in der Planung eingesetzt werden können. Die Beiträge aus Schweden (126) und Deutschland (520) widmeten sich dem Forecast von Mobilitätsladeprofilen sowie von Last- und Einspeiseprofilen auf Basis historischer Daten.

Im Kontrast dazu beschäftigte sich ein Beitrag aus Brasilien (895) mit der Erstellung von Lastprognosen in der Covid-19-Zeit.

26. Internationale Konferenz über elektrische Verteilnetze, virtuell, 20. bis 23. September 2021.

Braunstein, René, Energie Steiermark Technik GmbH, Leonhardgürtel 10, 8010 Graz, Österreich (E-Mail: rene.braunstein@e-steiermark.com) 
Auf Basis nicht vorhandener historischer Daten wurden unkonventionelle Al-Methoden in Kombination mit der Lastentwicklung in Europa und lokalem "Social Media Monitoring" angewendet.

Zusammenfassend können auf Basis der Beiträge 2021 folgende Tendenzen verzeichnet werden:

- Insbesondere die Beiträge aus dem Research and Innovation Forum (RIF), aber auch aus den Main-Sessions spiegeln den stärker werdenden Trend der Verschmelzung von Energietechnik und Informatik wider.

- Wie bei der letzten CIRED stellten die Erfassung, Verarbeitung und Analyse großer Datenmengen und auch die Prognose von
Einspeise- und Lastszenarien ein zentrales Thema dar. Daraus haben sich mittlerweile erste Konzepte und Projekte zur Flexibilität im Verteilernetz entwickelt.

- Das Thema der "Non-Wire Alternatives" (NWA) wurde aufs Tapet gebracht. Dafür müssen die regulatorischen Rahmenbedingungen neu geschaffen werden.

- Der Einsatz von DC-Elementen im Verteilernetz stand im Vergleich zur letzten CIRED weniger stark im Fokus der Session 5.

Hinweis des Verlags Der Verlag bleibt in Hinblick auf geografische Zuordnungen und Gebietsbezeichnungen in veröffentlichten Karten und Institutsadressen neutral. 